\title{
Acionamento de um Motor de Indução Trifásico com Incertezas Paramétricas e Falha no Atuador utilizando Controle Robusto com Taxa De Decaimento
}

\author{
Tiago V. Ortunho*, Marcelo C. M. Teixeira**, Jean M. S. Ribeiro**, Alexandre A. Carniato*, Leonardo A. Carniato*, \\ Diogo R. de Oliveira***. \\ *Instituto Federal de Educação, Ciência e Tecnologia de São Paulo - Câmpus Presidente Epitácio \\ Rua José Ramos Junior, 27-50 - Jardim Tropical - Presidente Epitácio - SP - 19470-000 \\ E-mails: tiago.veronese@ifsp.edu.br, carniato@ifsp.edu.br,leonardo@ifsp.edu.br \\ **Laboratório de Controle, Depto. de Engenharia Elétrica, Universidade Estadual Paulista Júlio de Mesquita Filho - \\ Campus Ilha Solteira \\ Avenida Brasil, 56 - Centro - Ilha Solteira - SP - 15385-000 \\ E-mails: marcelo.minhoto@unesp.br, jean.ribeiro@unesp.br \\ ***Instituto Federal de Educação, Ciência e Tecnologia de Mato Grosso do Sul - Câmpus Três Lagoas \\ Rua Ângelo Melão, 790 - Jardim das Paineiras - Três Lagoas - MS - 79641-162 \\ E-mails: diogo.ramalho@ifms.edu.br
}

\begin{abstract}
In this work, a robust controller with decay rate was designed for a three phase induction motor considering the model with parametric uncertainties (electric stator speed, rotor time constant and stator resistance). Later, using the same considerations aforementioned, a new controller was designed, taking into account faults in the actuator. The controller designs were developed considering access to all state variables and using Matlab/Simulink software with the Yalmip and Sedumi solvers. Through simulations it was possible to validate the developed control methodology.

Resumo: Neste trabalho foi projetado um controlador robusto com taxa de decaimento para um motor de indução trifásico considerando o modelo com incertezas paramétricas (velocidade elétrica do estator, constante de tempo do rotor e resistência do estator). Posteriormente, utilizando as mesmas considerações apresentadas, foi projetado um novo controlador levando-se em conta uma falha no atuador. Os projetos dos controladores foram desenvolvidos considerando acesso a todas as variáveis de estado e utilizando o software Matlab/Simulink com os solvers Yalmip e Sedumi. Através de simulações foi possível validar a metodologia de controle desenvolvida.
\end{abstract}

Keywords: Linear Matrix Inequalities; Parametric Uncertainties; Three Phase Induction Motor.

Palavras-chaves: Desigualdade Matriciais Lineares; Incertezas paramétricas; Motor de Indução Trifásico.

\section{INTRODUÇÃO}

O motor de indução trifásico (MIT) é muito utilizado industrialmente e, devido às diversas pesquisas desenvolvidas aumenta-se a sua aplicabilidade tendo em vista as suas vantagens comparativamente a outras máquinas, principalmente relacionado com sua robustez.

Mesmo sendo robusto ocorrem falhas e quebras. Segundo Dionysios e Epaminondas (2013), 44\% das falhas estão relacionadas ao rolamento, $26 \%$ devido ao estator, $8 \%$ ao rotor e $22 \%$ por outros motivos.

Além das falhas que ocorrem no equipamento um dos principais problemas relacionados às metodologias de controle do equipamento são provenientes das variações paramétricas da máquina.

Em Marino, Tomei e Verrelli (2008) uma variação de $20 \%$ na resistência do estator pode tornar o sistema controlado instável.
Diante desses fatos, esse trabalho abordou o projeto de controladores robustos com taxa de decaimento considerando incertezas e falha no sinal do atuador. Através de simulações foi comprovada a metodologia de controle adotada.

\section{MODELO DO MOTOR DE INDUÇÃO TRIFÁSICO}

As equações dinâmicas da máquina de indução trifásica podem ser encontradas na literatura com Bose (2001), Krishman (2001), Palma (1999) e, através destas, é possível definir o modelo em espaço de estados (3) do motor de indução trifásico com rotor gaiola de esquilo no sistema de referência síncrona girante com orientação pelo fluxo do rotor (1) e (2):

$$
\phi_{q r}^{e}=\frac{d}{d t} \phi_{q r}^{e}=0
$$




$$
\phi_{r}=\phi_{d r}^{e}=\phi_{d r}^{e^{*}}=\text { constante. }
$$

Na representação em espaço de estados adotou-se o torque de carga como perturbação do sistema:

$$
\begin{aligned}
& \dot{x}(t)=A(\alpha) x(t)+B u(t)+B_{W} T_{L}(t), \\
& A(\alpha)=\sum_{i=1}^{r} \alpha_{i} A_{i}, \alpha_{i} \in[0,1], \sum_{i=1}^{r} \alpha_{i}=1
\end{aligned}
$$

em que $x(t) \epsilon R^{n}$ é o vetor de estado do sistema, $u(t) \epsilon R^{m}$ é o sinal de controle, $B \in R^{n x m}$ é a matriz de entrada, $A(\alpha)$ é a matriz do sistema, $\operatorname{com} A_{i} \in R^{n x n}$ e $i \epsilon K_{r}=\{1,2, \ldots, r\}$, no qual, $r \in N$ e $r=2^{s}$, sendo que a variável $s$ representa o número de parâmetros incertos e/ou falhas presentes no sistema.

As variáveis apresentadas em (3) são descritas em (4) - (8).

$$
\begin{aligned}
& A(\alpha)=\left[\begin{array}{llll}
a_{11} & a_{12} & a_{13} & a_{14} \\
a_{21} & a_{22} & a_{23} & a_{24} \\
a_{31} & a_{32} & a_{33} & a_{34} \\
a_{41} & a_{42} & a_{43} & a_{44}
\end{array}\right] ; \\
& a_{11}=-\left[\frac{R_{s}}{\sigma L_{s}}+\frac{(1-\sigma)}{\sigma \tau_{r}}\right] ; a_{12}=w_{e} \text {; } \\
& a_{13}=\frac{L_{m}}{\sigma L_{s} L_{r} \tau_{r}} ; a_{21}=-w_{e} ; a_{22}=-\frac{R_{s}}{\sigma L_{s}} ; \\
& a_{14}=a_{24}=a_{32}=a_{34}=a_{41}=a_{43}=0 \text {; } \\
& a_{23}=-w_{e} \frac{L_{m}}{\sigma L_{s} R_{r} \tau_{r}} ; a_{31}=\frac{L_{m}}{\tau_{r}} ; \\
& a_{33}=-\frac{1}{\tau_{r}} ; a_{42}=\frac{K_{t}}{J} ; a_{44}=-\frac{B}{J} ; \\
& B=\left[\begin{array}{ll}
b_{11} & b_{12} \\
b_{21} & b_{22} \\
b_{31} & b_{32} \\
b_{41} & b_{42}
\end{array}\right] ; b_{11}=b_{22}=\frac{1}{\sigma L_{s}} ; \\
& b_{12}=b_{31}=b_{32}=b_{41}=b_{42}=0 \text {; } \\
& B_{w}=\left[\begin{array}{llll}
0 & 0 & 0 & -\frac{1}{J}
\end{array}\right]^{T} \\
& x(t)=\left[\begin{array}{llll}
i_{d s}^{e} & i_{q s}^{e} & \phi_{d r}^{e} & w_{r}
\end{array}\right]^{T} ; \\
& u(t)=\left[\begin{array}{ll}
V_{d s}^{e} & V_{q s}^{e}
\end{array}\right]^{T} ;
\end{aligned}
$$

sendo que:

$R_{s} ; R_{r} \rightarrow$ resistências do estator e do rotor;

$L_{s} ; L_{r} ; L_{m} \rightarrow$ indutâncias do estator, do rotor e mútua; $\sigma \rightarrow$ coeficiente de dispersão; $w_{e} ; w_{r} \rightarrow$ velocidade elétrica do estator e velocidade do rotor; $J \rightarrow$ momento de inércia;

$B \rightarrow$ coeficiente de atrito viscoso;

$p \rightarrow$ número de pares de pólos;

$i_{d s}{ }^{e} ; i_{q s}{ }^{e} \rightarrow$ correntes do estator no eixo direto e em quadratura na referência síncrona girante;

$V_{d s}{ }^{e} ; V_{q s}{ }^{e} \rightarrow$ tensões do estator no eixo direto e em quadratura na referência síncrona girante;

$\phi_{d r}{ }^{e} ; \phi_{q r}{ }^{e} \rightarrow$ fluxos do rotor no eixo direto e quadratura na referência síncrona girante;

$T_{e} \rightarrow$ torque eletromagnético;

$T_{L} \rightarrow$ torque de carga;

$\tau_{r} \rightarrow$ constante de tempo do rotor;

$K_{t} \rightarrow$ constante de torque.

Adicionalmente, o coeficiente de dispersão, a constante de tempo do rotor e a constante de torque são apresentados de (9) a (11):

$$
\begin{gathered}
\sigma=1-\frac{L_{m}^{2}}{L_{s} L_{r}}, \\
\tau_{r}=\frac{L_{r}}{R_{r}}, \\
T_{e}=\frac{3 p}{2} \frac{L_{m}^{2}}{L_{r}} i_{d s}^{e^{*}} i_{q s}^{e}=\frac{3 p}{2} \frac{L_{m}}{L_{r}} \phi_{d r}^{e^{*}} i_{q s}^{e}=K_{t} i_{q s}^{e} .
\end{gathered}
$$

\section{CONTROLE ROBUSTO COM TAXA DE DECAIMENTO VIA LMI}

A metodologia de controle utilizada neste trabalho supõe que todas as variáveis de estado do sistema (3) estão disponíveis para realimentação.

Considerando a lei de controle (12) e o Teorema 1, desenvolveu-se o sistema de controle, com taxa de decaimento $\delta$.

$$
u(t)=-K x(t), K \in R^{m x n} .
$$

Teorema 1: (BOYD et al., 1994), o sistema (3) será estável e com taxa de decaimento maior ou igual a $\delta$ se existir um escalar $\delta>0$, uma matriz $Z \in R^{m x n}$ e uma matriz simétrica positiva definida $W \in R^{n x n}$ tal que (13).

$$
\begin{aligned}
& W A(\alpha)^{T}-Z^{T} B^{T}+A(\alpha) W-B Z+2 \delta W<0, \\
& W>0 .
\end{aligned}
$$

Com a LMI (13) é possível controlar o sistema obtendo respostas rápidas, através da definição da constante positiva $\delta$.

$\mathrm{O}$ ajuste adequado da taxa de decaimento possibilita a especificação da rapidez da resposta dos atuadores para o controle do sistema, pois, ela respeita a expressão (14): 


$$
\lim _{t \rightarrow \infty} e^{\delta t}\|x(t)\|=0
$$

A definição do controlador que atende ao critério de desempenho estabelecido pelo projetista e torna o sistema controlado estável é calculado através de (15):

$$
K=Z W^{-1}
$$

O Teorema 1 é comprovado efetuando-se um estudo da estabilidade, utilizando-se uma função quadrática de Lyapunov, para o sistema realimentado.

Para um acionamento adequado do motor de indução trifásico, é necessário utilizar sinais de referência para o fluxo do eixo direto do rotor e para a velocidade do rotor (16):

$$
r(t)=\left[\begin{array}{c}
\phi_{d r}^{e^{*}} \\
w_{r}^{*}
\end{array}\right]
$$

Visando manter o erro de seguimento de referência nulo expandiram-se as matrizes do sistema das variáveis de estado conforme proposto em Ogata (1998). O erro de regime é representado em (17):

$$
e(t)=\left[\begin{array}{c}
\int_{0}^{t}\left(\phi_{d r}^{e}(t)-\phi_{d r}^{e^{*}}(t)\right) d t \\
\int_{0}^{t}\left(w_{r}(t)-w_{r}^{*}(t)\right) d t
\end{array}\right]
$$

\section{PROJETO E RESULTADOS DE SIMULAÇÕES PARA O ACIONAMENTO DO MIT COM INCERTEZAS POLITÓPICAS}

O projeto dos controladores para o acionamento do motor de indução trifásico com potência nominal de $728 \mathrm{~W}, 2000$ RPM, $130 \mathrm{~V}$ e 5,6 A foi desenvolvido utilizando os dados da Tabela 1. Estes foram utilizados no trabalho Chang et al. (2005).

A partir dos dados da Tabela 1 foram desenvolvidos dois projetos para o controle do MIT.

Primeiramente, considerou-se que o motor de indução trifásico possui incertezas na velocidade elétrica do estator, sendo na sua regra de operação, $w_{\text {emax }}=510(\mathrm{rad} / \mathrm{s})$ e $w_{\text {emin }}=$ $-510(\mathrm{rad} / \mathrm{s})$. Além desta incerteza, considerou-se a constante de tempo do rotor e a resistência elétrica do estator como parâmetros variantes, pois sofrem influência da temperatura, tempo de operação, saturação magnética, sujeira, sobrecarga e envelhecimento do material, além do que, estes parâmetros também se modificam por falhas no equipamento, como, curto circuito entre espiras, curto circuito entre bobinas, quebra de barras do rotor e quebra do anel de curto circuito do rotor.

Adotou-se que a faixa de variação dos parâmetros é de $20 \%$, ou seja, $\tau_{r \max }=1,1 \tau_{r}[\mathrm{~s}], \tau_{\text {rmin }}=0,9 \tau_{r}[\mathrm{~s}]$ e $R_{\text {smax }}=1,1 R_{s}[\Omega] \mathrm{e}$ $R_{\text {smin }}=0,9 R_{s}[\Omega]$. A quantidade de incertezas consideradas em uma mesma análise representa a contribuição do trabalho comparativamente à literatura existente.

Tabela 1. Dados do motor de indução trifásico.

\begin{tabular}{|c|c|}
\hline Dados & Valores \\
\hline Resistência do estator $\left(R_{s}\right)$ & $629 \mathrm{~m}[\Omega]$ \\
\hline Resistência do rotor $\left(R_{r}\right)$ & $775 \mathrm{~m}[\Omega]$ \\
\hline Indutância do estator $\left(L_{s}\right)$ & $68,92 \mathrm{~m}[\mathrm{H}]$ \\
\hline Indutância do rotor $\left(L_{r}\right)$ & $68,92 \mathrm{~m}[\mathrm{H}]$ \\
\hline Indutância mútua $\left(L_{m}\right)$ & $65,47 \mathrm{~m}[\mathrm{H}]$ \\
\hline Pares de polo do motor $(p)$ & 2 \\
\hline Momento de inércia $(J)$ & $4,78 \mathrm{~m}\left[\mathrm{~kg} . \mathrm{m}^{2}\right]$ \\
\hline Coeficiente de atrito viscoso $(B)$ & $5,34 \mathrm{~m}[\mathrm{~m} / \mathrm{rad} . \mathrm{s}]$ \\
\hline Fluxo nominal do rotor $\left(\phi_{d r}{ }^{e}\right)$ & $0,1[\mathrm{~Wb}]$ \\
\hline Torque de carga $\left(T_{L}\right)$ & $0,8[\mathrm{~N} . \mathrm{m}]$ \\
\hline Coeficiente de dispersão $(\sigma)$ & $97,6103 \mathrm{~m}$ \\
\hline
\end{tabular}

O controlador robusto projetado adotou a especificação de taxa de decaimento $\delta=10$ e utilizando o software Matlab e os solvers YALMIP e SEDUMI foi desenvolvido o projeto obtendo (18):

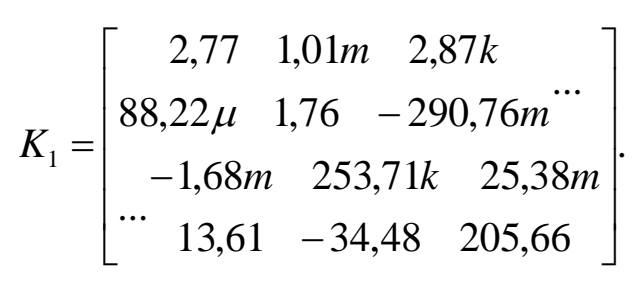

Em seguida, objetivando obter um controlador que atenda aos mesmos critérios desenvolvidos anteriormente, mas que também, possibilite abranger falha no sistema de potência, como por exemplo, falta de fase, danos ao barramento cc e perdas excessivas nos dispositivos semicondutores (diodos retificadores e/ou IGBT's) considerou-se uma falha de $10 \%$ no sinal de atuação, assim sendo, (5) foi substituída por (19):

$$
\begin{aligned}
& B=\left[\begin{array}{ll}
b_{11} & b_{12} \\
b_{21} & b_{22} \\
b_{31} & b_{32} \\
b_{41} & b_{42}
\end{array}\right] ; b_{11}=b_{22}=\frac{b}{\sigma L_{s}} ; \\
& b_{12}=b_{31}=b_{32}=b_{41}=b_{42}=0 .
\end{aligned}
$$

Considerando que a faixa de operação da incógnita $b$ é $b_{\max }=$ 1 e $b_{\min }=0,9$, obteve-se o controlador (20).

O comportamento de ambos controladores foram analisados através do software Matlab/Simulink.

A simulação foi desenvolvida considerando o sinal de referência de velocidade constante e no instante de tempo de 15 segundos aplicou-se um degrau de carga com torque nominal no sistema. 


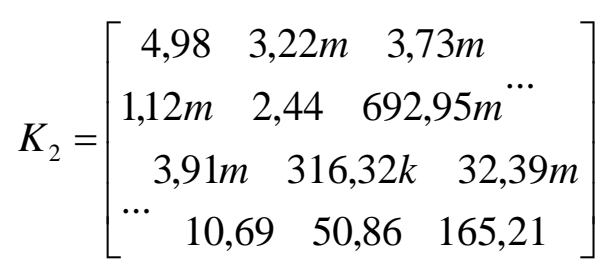

A seguir, são apresentados os resultados do comportamento do MIT utilizando o controlador $K_{l}$.

Considerando o sistema com três incertezas paramétricas e/ou falhas, obteve-se um conjunto com oito vértices no politopo.

Para evitar um excesso de informações optou-se por apresentar as respostas para dois vértices, sendo estes, o vértice um que possui as incógnitas com valores mínimos e o vértice oito que possui os valores máximos.

Portanto, na Figura 1 é apresentada a resposta da velocidade para ambos os vértices sendo a velocidade de referência considerada no valor de $210 \mathrm{rad} / \mathrm{s}$ (nominal).

Para uma melhor visualização, mostra-se o instante de partida e o momento da aplicação do degrau de carga do motor.

A velocidade demorou aproximadamente 0,3 segundos para atingir o valor de referência e no momento da aplicação do degrau de carga este tempo foi de 0,2 segundos. A oscilação da velocidade durante a aplicação da carga foi menor que 1 $\mathrm{rad} / \mathrm{s}$.

Na Figura 2 são mostrados os sinais do fluxo do rotor para os mesmos vértices adotados anteriormente. Note que o sinal de referência do fluxo do rotor é de $0,1 \mathrm{~Wb}$, um zoom foi desenvolvido nos momentos da partida e na aplicação da carga.
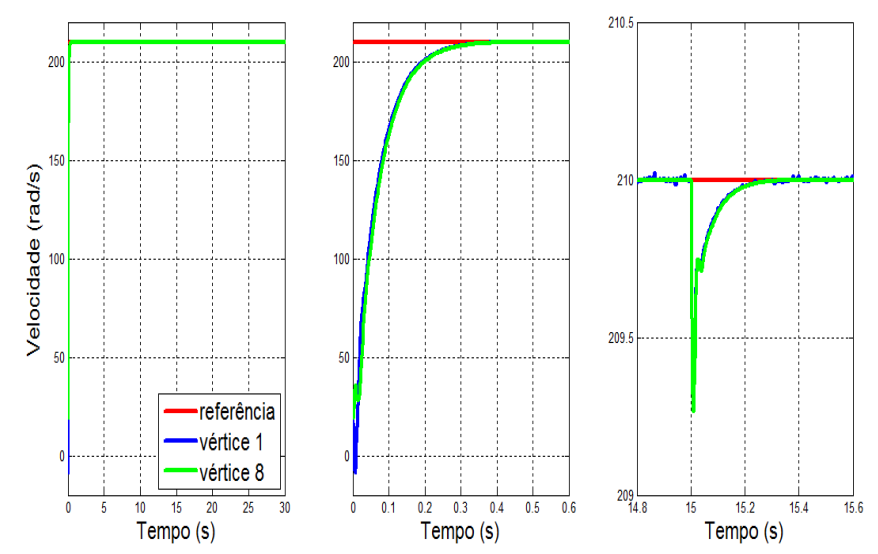

Fig. 1 Velocidade do motor com referência constante.

Verifica-se que a velocidade e o fluxo do rotor acompanharam o sinal de referência para o sistema incerto e, mesmo no instante de aplicação do degrau de carga, os sinais rapidamente reduziram o erro de regime.

Nas Figuras 3 e 4 são apresentados os comportamentos das correntes do eixo direto e do eixo de quadratura do MIT. Da mesma forma, que apresentado anteriormente, efetuou-se um zoom nos momentos da partida e da aplicação do degrau de carga.

Nas Figuras 5 e 6 são apresentadas as tensões do eixo direto e do eixo de quadratura, considerando a referência de velocidade constante com o motor sem carga e em 15 segundos aplicando o degrau nominal de carga.
Evidencia-se que nas Figuras de 3 a 6 as correntes e as tensões tiveram bons resultados mantendo-se dentro de níveis aceitáveis para implementação. Apenas, no momento de partida do motor, os valores ficaram elevados, sendo necessário utilizar alguma metodologia para evitar a sobretensão no equipamento.

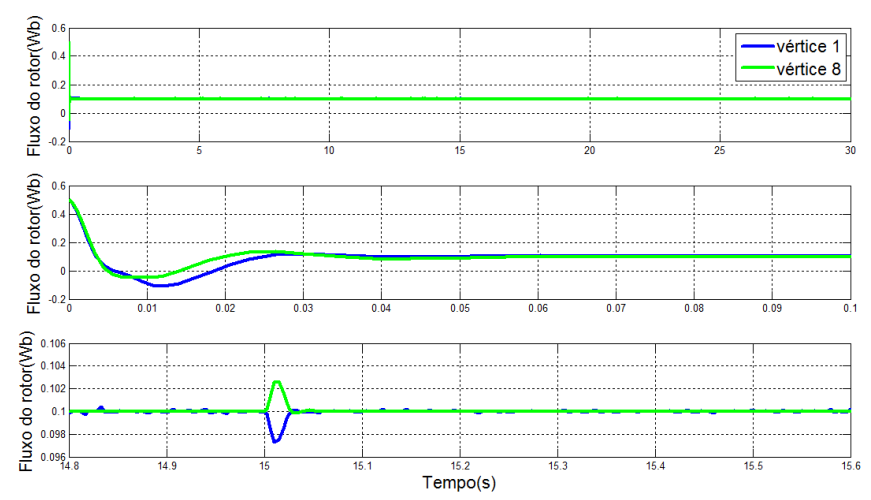

Fig. 2 Fluxo do rotor com velocidade constante.
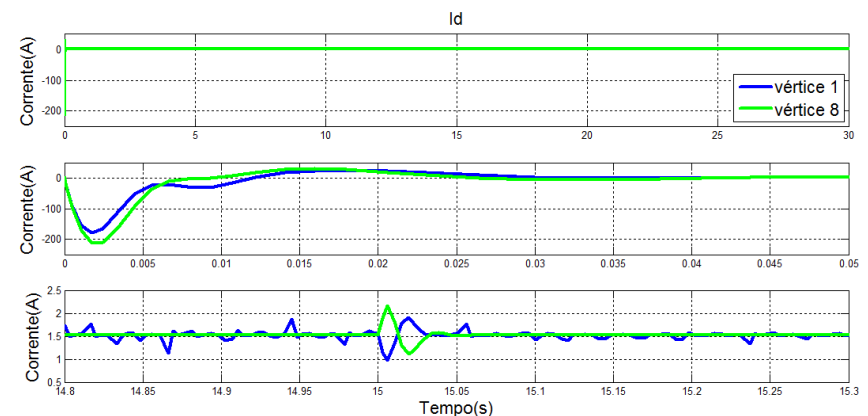

Fig. 3 Corrente do eixo direto com velocidade constante.
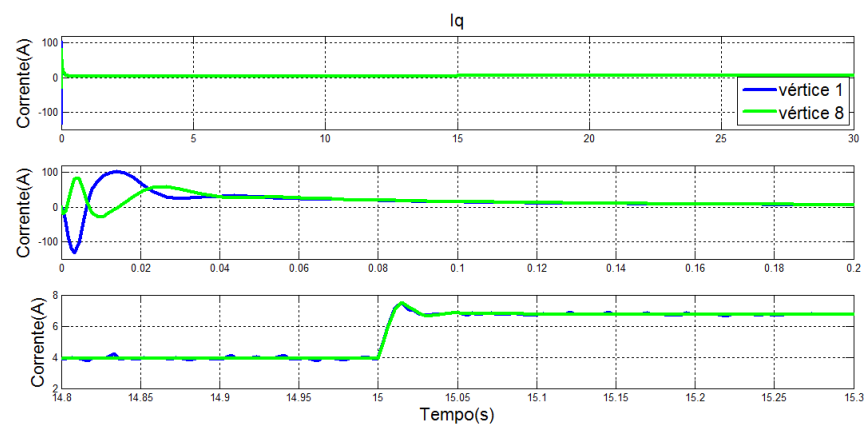

Fig. 4 Corrente do eixo de quadratura com velocidade constante.

A seguir, são apresentados os resultados de simulação do sistema considerando o uso do controlador $K_{2}$, no qual se considerou as mesmas condições utilizadas para o controlador $K_{l}$, diferindo apenas que agora o sistema possui uma falha no sinal de controle.

A falha foi aplicada no tempo de 7 a 13 segundos e ocorreu novamente na faixa de 21 a 27 segundos.

Na Figura 7 é apresentado o desempenho do sistema para uma referência de velocidade constante em $210 \mathrm{rad} / \mathrm{s}$, com incertezas, falha na atuação do controlador e torque nominal. Nela efetuou-se um zoom no instante de partida no centro superior, no momento da aplicação do torque nominal superior direito, no canto inferior esquerdo retirou-se o 
momento da partida para uma melhor visualização das magnitudes atingidas e, por fim, no centro e no canto direito inferior verifica-se, com mais detalhes, os instantes de falha dos atuadores.
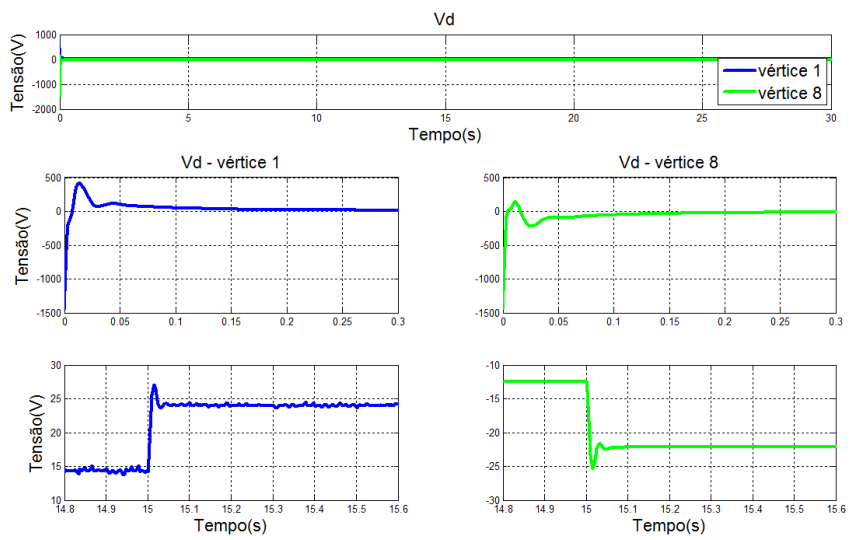

Fig. 5 Tensão do eixo direto com velocidade constante.
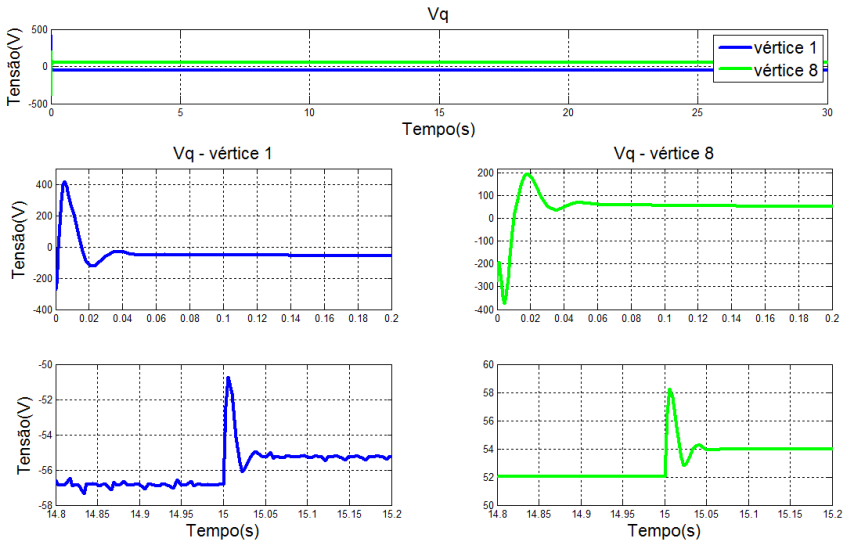

Fig. 6 Tensão do eixo de quadratura com velocidade constante.
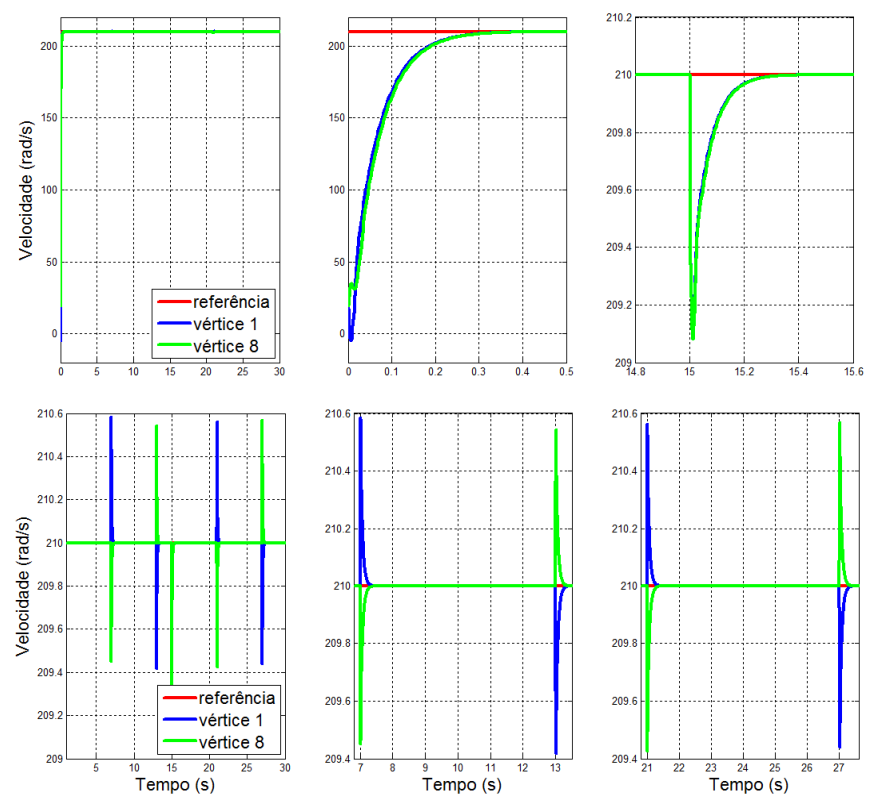

Fig. 7 Velocidade do motor com referência constante e falha no atuador.
Na Figura 8 tem-se o comportamento do fluxo magnético do rotor para as condições analisadas, evidenciando os momentos de partida, aplicação do torque e falha no atuador. Nas Figuras 9 e 10 apresenta-se o comportamento das correntes de eixo direto e de quadratura.
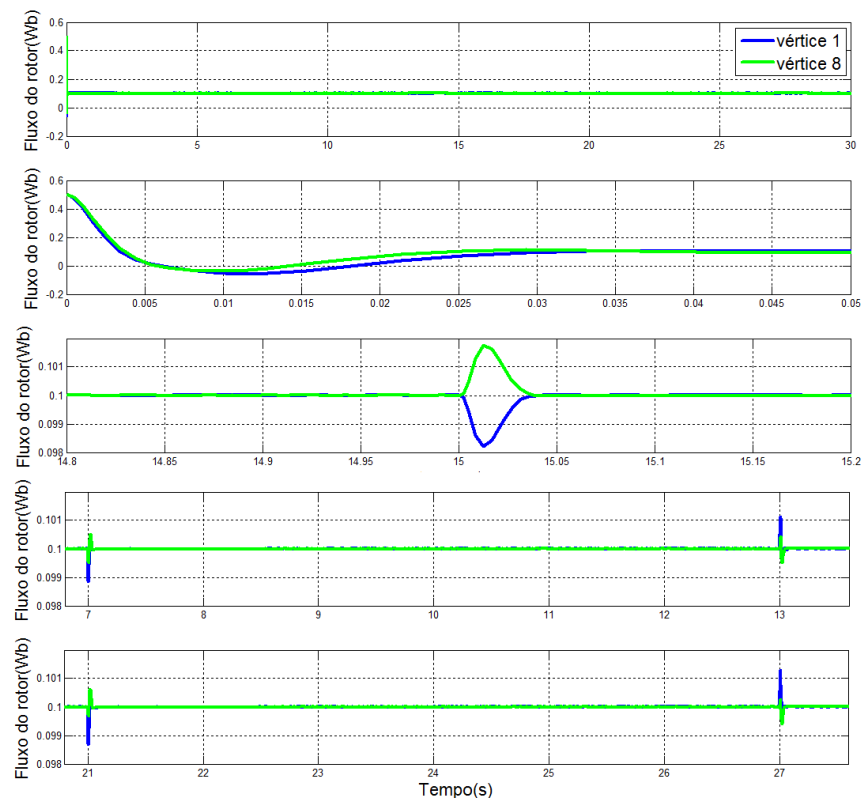

Fig. 8 Fluxo do rotor com velocidade constante e falha no atuador.
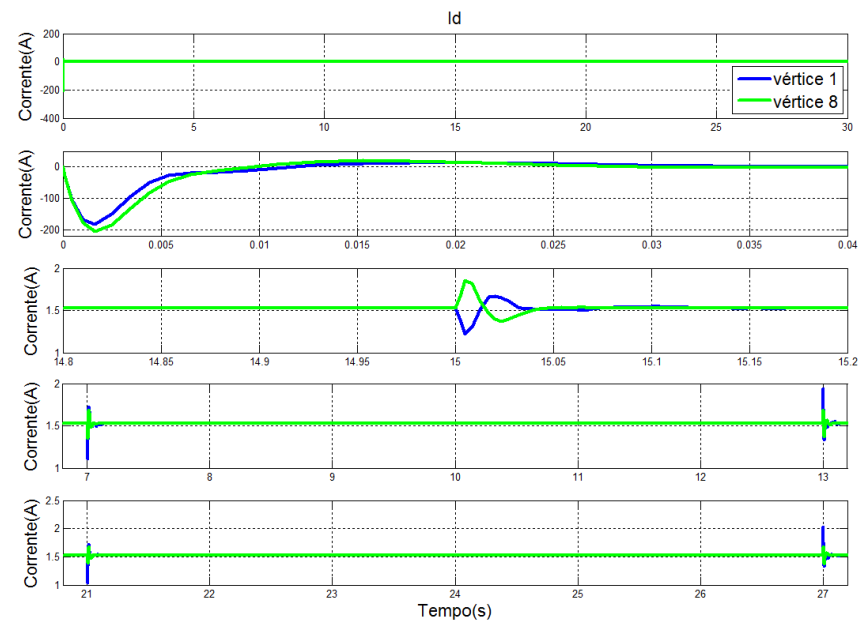

Fig. 9 Corrente do eixo direto com velocidade constante e falha no atuador.

Através das simulações desenvolvidas pode-se afirmar que a utilização da metodologia de controle com realimentação de estados com taxa de decaimento via LMIs possibilitou um bom controle para o acionamento do MIT considerando três tipos de incertezas e falha no atuador.

\section{CONCLUSÕES}

O trabalho desenvolvido apresenta o projeto de dois controladores robustos com taxa de decaimento utilizando desigualdades matriciais lineares para o acionamento de um motor de indução trifásico com incertezas paramétricas, sendo elas, na velocidade elétrica do estator, na constante de 
tempo do rotor e na resistência do estator. Além disto, um dos projetos considerou o sistema com falha no atuador.
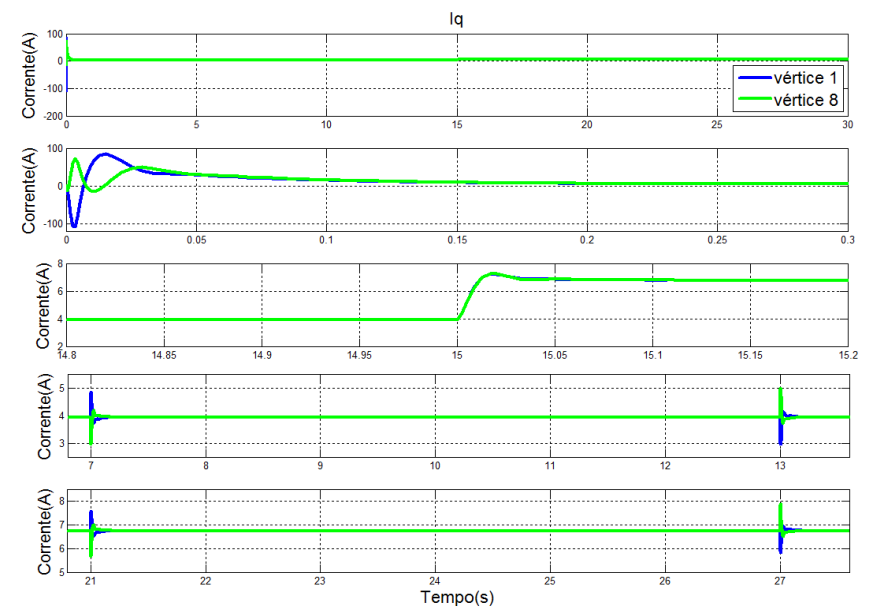

Fig 10 Corrente do eixo de quadratura com velocidade constante e falha no atuador.

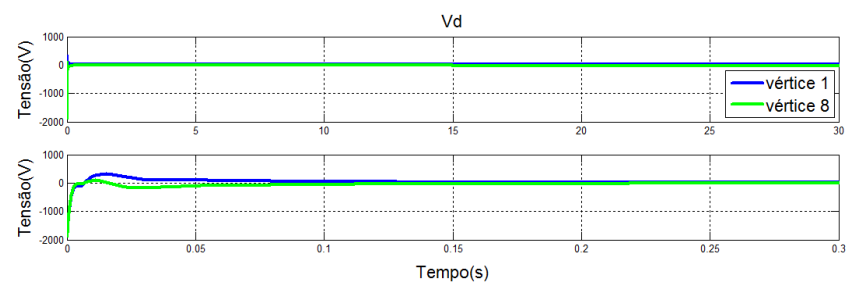

Fig. 11 Tensão do eixo direto com velocidade constante e falha no atuador.
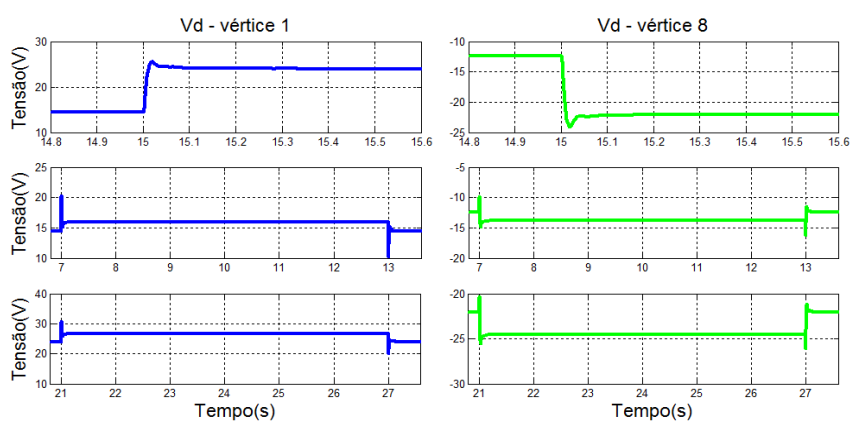

Fig. 12 Zoom na tensão do eixo direto com velocidade constante e falha no atuador.
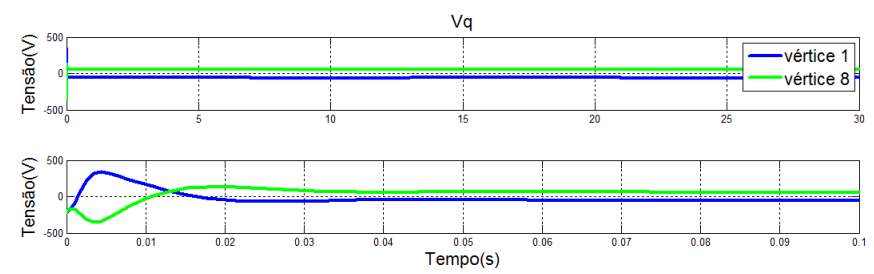

Fig. 13 Tensão do eixo de quadratura com velocidade constante e falha no atuador.

O projeto consistiu em encontrar ganhos do controlador que estabiliza o sistema atendendo ao tempo de estabelecimento definido em projeto.

A consideração de incertezas paramétricas possibilita a aplicação dos controladores projetados em processos industriais do MIT, pois, durante a operação do equipamento, condições do ambiente, carga acionada e outras variáveis acarretam a modificação de seus parâmetros ocasionando problemas na dinâmica do sistema.
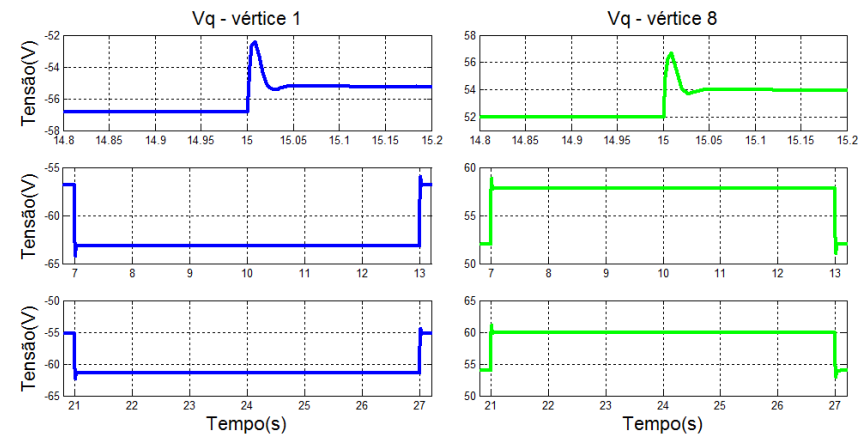

Fig. 14 Zoom na tensão do eixo de quadratura com velocidade constante e falha no atuador.

Através de simulações com o software Matlab/Simulink foi verificada a eficiência do controle proposto.

Ambas as condições analisadas tiveram bom comportamento, apenas no momento de partida devem-se utilizar metodologias para reduzir a tensão de controle objetivando manter a magnitude do sinal dentro da faixa tolerada do acionamento ou utilizar outros artifícios para melhorar a tipologia estudada.

O trabalho forneceu uma aplicação importante para a metodologia de controle e a continuidade da pesquisa proporcionará uma melhoria no acionamento do MIT.

\section{AGRADECIMENTOS}

Os autores agradecem a UNESP/FEIS, o IFSP/PEP e a CAPES por possibilitarem o desenvolvimento da pesquisa.

\section{REFERÊNCIAS}

Bose, B.K. (2001). Modern power electronics and ac drives. Prentice Hall, Upper Saddle River.

Boyd, S., Ghaoui, L.E., Feron, E., and Balakrishnan, V. (1994). Linear matriz inequalities in systems and control theory. SIAM Studies in Applied Mathematics, Philadelphia.

Chang, Y., Wang, Y., Hung, M., and Chen, P. (2005). Regional stability and $\mathrm{H} \infty$ performance control of an input satured induction motor via LMI approach. Asian Journal of Control, volume (7), 12.

Dionysios, V.S., and Epaminondas, D.M. (2013). A review on the faults of electric machines used in electric ships. Advances in Power Electronics, volume (2013), 8.

Krishman, R. (2001). Electric motor drives modeling, analysis and control. Upper Saddle, New Jersey.

Marino, R., Tomei, P., and Verrelli, C.M. (2008). An adaptive tracking control from current measurements for induction motors with uncertain load torque and rotor resistance. Automatica, volume (44), 6.

Ogata, K. (1998). Engenharia de controle moderno. LTC, Rio de Janeiro.

Palma, J.C.P. (1999). Accionamentos electromecânicos de velocidade variável. Fundação Calouste Gulbenkian, Lisboa. 